\title{
Kooperation zwischen der FOM Fachhochschule für Oekonomie \& Management und der List Gesellschaft e.V.
}

Auf der außerordentlichen Mitgliederversammlung der List Gesellschaft am 4 . Dezember 2008 wurde eine Kooperationsvereinbarung mit der FOM Fachhochschule für Oekonomie \& Management (FOM) für eine Laufzeit von zunächst drei Jahren beschlossen. Gegenstand der am 1. Januar 2009 begonnenen Kooperation ist das gemeinsame Ziel der Partner, den Dialog zwischen Wissenschaft und Praxis über wirtschafts- und gesellschaftspolitische Themen, beispielsweise durch gemeinsame Veranstaltungen und Publikationen, zu fördern.

In Folge der Kooperation ist die Geschäftsstelle der List Gesellschaft seit dem 1. Januar 2009 an der FOM in Essen angesiedelt und wird durch Herrn Dipl.-Jur. Kai E. Stumpp betreut. Die neuen Kontaktdaten der Geschäftsstelle lauten:

\section{List Gesellschaft e.V.}

FOM Fachhochschule für Oekonomie \& Management

Leimkugelstraße 6, 45141 Essen

Telefon: +49 (0) $201-240967$ 544, Telefax: +49 (0) $201-240967399$

E-Mail: mail@list-gesellschaft.de, Internet: www.list-gesellschaft.de

Die FOM versteht sich als Hochschule der Wirtschaft für Berufstätige - und damit als Ergänzung zur deutschen Hochschullandschaft. Sie bereitet durch anwendungsbezogene, berufs- und ausbildungsbegleitende Bachelor- und Master-Studiengänge auf leitende bzw. unternehmerische Tätigkeiten vor. Ihre Studiengänge werden in Präsenzform angeboten und zielen auf den Ausbau und den Erhalt der Beschäftigungsfähigkeit im Kontext lebenslangen Lernens und beruflicher Kompetenzentwicklungsprozesse ab.

Derzeit studieren an der FOM über 15.000 Berufstätige und Auszubildende an bundesweit 19 Standorten und in Luxemburg parallel zu ihrer betrieblichen Tätigkeit abends und am Wochenende.

\section{Weitere Informationen zur FOM:}

FOM Fachhochschule für Oekonomie \& Management gGmbH

Leimkugelstraße 6, 45141 Essen

Tel.: $01801-810048$, Fax: $01801-810049$ (bundesweit zum City-Tarif)

Email: studienberatung@fom.de, Internet: www.fom.de 